\title{
A WATER-POWERED MICRO DRUG DELIVERY SYSTEM
}

\author{
Yu-Chuan Su, Liwei Lin, and Albert P. Pisano \\ Department of Mechanical Engineering and Berkeley Sensor \& Actuator Center \\ University of California at Berkeley \\ Berkeley, CA 94720-1740
}

\begin{abstract}
This paper presents a plastic micro drug delivery system that draws power directly from water without using any electrical power and delivers liquid drugs with pressure up to $25 \mathrm{MPa}$ to overcome possible blockages from microorganisms. The system consists of an osmotic micro-actuator, a drug reservoir, a microfluidic channel to control the diffusive flow of drug, and a drug delivery port. The actuating membrane has an initial diameter of $800 \mu \mathrm{m}$ and the length of the microfluidic channel is $1 \mathrm{~cm}$ with a cross section of $30 \times 100 \mu \mathrm{m}^{2}$. Using oxygen plasma to activate polymer surfaces, simultaneous sealing (in the presence of water) and encapsulation of liquid are accomplished. Employing the net water flow induced by osmosis, this prototype drug delivery system has a measured, constant delivery rate at $0.2 \mu \mathrm{l} / \mathrm{hr}$ for an operation period of 10 hours with a delivery volume of $2 \mu 1$. The delivery rate and volume can be designed to target specific disease for the treatment period of hours up to years. As such, this micro drug delivery system has strong potential in biomedical applications.
\end{abstract}

\section{INTRODUCTION}

In recent years, MEMS (Microelectromechanical Systems) techniques have been applied to a variety of medical research to both improve the performance of existing devices and to explore new territories that realized by the advanced micro-machining technologies. Drug delivery, which covers a broad range of techniques for transporting therapeutic agents into the human body, remains an important challenge in medicine and the applications of MEMS techniques may have open up new research opportunities.

Implantable, controlled-release drug delivery systems offer benefits over repetitive administration of conventional drug therapy by providing unattended continuous delivery within the therapeutic window. Avoiding highly variable peak concentrations often seen after immediate release of doses, constant drug concentration delivery systems as the one presented in this paper can result in enhanced drug efficacy and minimized side effects [1]. Furthermore, these systems have the potential to provide alternative paths to deliver special drug components such as proteins and peptides, which are often difficult to administrate due to rapid degradation and poor absorption in the gastrointestinal tract. Another example is macromolecule, which is difficult to deliver by other techniques [2].

Osmotic drug delivery systems have been used for both oral and implantable constant-rate drug delivery for decades [3]. Previously, we have demonstrated an osmotic micro-actuator by means of microfabrication [4]. This work presents the advances in an all-plastic, micro drug delivery system. Three technical accomplishments have been achieved in this work: (1) bonding of PDMS with barrier polymers by introducing an intermediate elastomer layer for system integration; (2) direct liquid encapsulation and sealing at room temperature in the presence of water; and (3) delivery rates up to 20 times faster than previous achieved in microscale osmotic actuation. Powered by osmosis, this micro drug delivery system has the potential as an implantable drug delivery system to provide constant-rate drug release profiles.

\section{PRINCIPLES AND THEORETICAL MODELS}

A schematic diagram of the micro drug delivery system is illustrated in Figure 1. The system is composed of two major parts: the osmotic actuator at the bottom and microfluidic components including the drug storage reservoir and the delivery channel at the top. The details of the micro osmotic actuator have been reported previously [4] while the top part is made of Polydimethylsiloxane (PDMS) to form the drug reservoir and the delivery channel. Induced by the concentration difference across the semipermeable membrane at the bottom of the osmotic actuator, water from aqueous environment is drawn through the semipermeable membrane into the chamber filled with osmotic driving agent to power the drug delivery system. By virtue of the incompressibility of water, the top actuating membrane, which is hundreds of times more flexible than the bottom semipermeable membrane, expands to drive and release the liquid drug stored in the reservoir. The convective flow rate of drug driven out of the reservoir can be represented as follows:

$$
Q_{d c}=C_{d} \cdot Q_{c}
$$

where $Q_{d c}$ is the convective flow rate of drug, $C_{d}$ is the concentration of drug in the formulation within reservoir, and $Q_{c}$ is the overall convective flow rate of drug and its diluents, which is equal to the expansion rate of the actuating membrane.

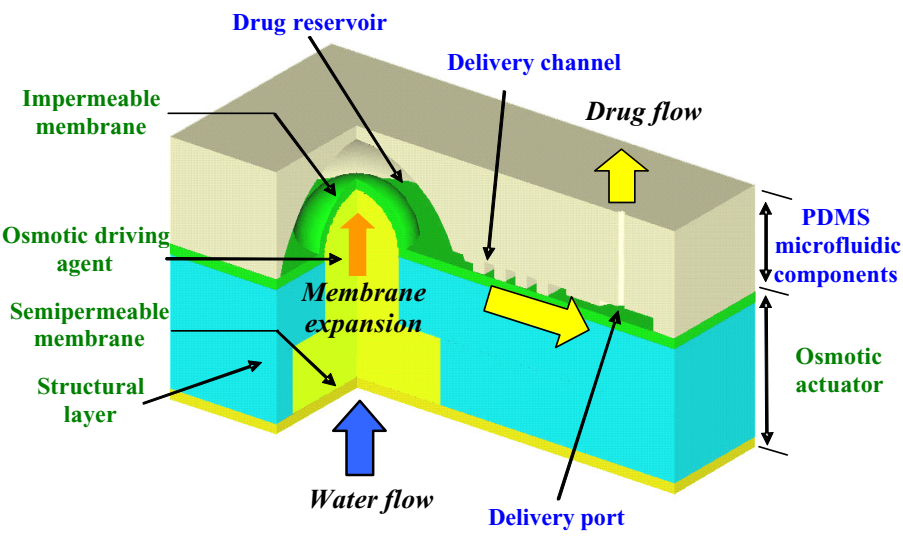

Figure 1. A schematic diagram of the micro drug delivery system. The integration of PDMS microfluidic components and osmotic actuator is achieved by plasma-activated bonding.

In addition to convective flow, drug is also released to outside environment by diffusion. The diffusive flow rate of drug through the delivery channel can be simplified as follows:

$$
Q_{d d}=D A \Delta C_{d} / L
$$

where $Q_{d d}$ is the diffusive flow rate of drug, $D$ is the diffusivity of drug through the delivery channel, $A$ is the cross-sectional area of the delivery channel, $\Delta C_{d}$ is the difference of drug concentration between inside reservoir and outside environment, and $L$ is the length of delivery channel. Generally, the drug concentration in 
the inside reservoir is much higher than the drug concentration in the outside environment such that $\Delta C_{d}$ can be approximated by $C_{d}$. It is desired to keep the diffusive flow rate of drug much less than the convective flow rate of drug such that the release rate of drug will be constant and controlled by osmosis. A performance index can be defined as follows [2]:

$$
I_{c}=\frac{Q_{d d}}{Q_{d c}}=\frac{D A}{Q_{c} L}
$$

where $I_{c}$ is the ratio of diffusive flow rate to convective flow rate of drug, which defines the consistency of drug flow and lower values indicate better consistency. With specified drug diffusivity and delivery rate, smaller cross-sectional area and longer length of the delivery channel will improve the consistency but also increase the pressure drop across the delivery channel, which can be represented as follows [5]:

$$
\Delta P=\frac{12 Q_{c} \mu L}{w h^{3}}\left[1-\frac{h}{w}\left(\frac{192}{\pi^{5}} \sum_{n=1}^{\infty} \frac{1}{n^{5}} \tanh \left(\frac{n \pi w}{h}\right)\right)\right]^{-1}
$$

where $\Delta P$ is the pressure drop across the delivery channel, $\mu$ is the viscosity of the formulation within reservoir, $w$ is the width of the delivery channel, and $h$ is the height of the delivery channel. The pressure drop should be kept under less than $10 \%$ of the driving pressure the actuator can provide to assure normal operation of the overall system.
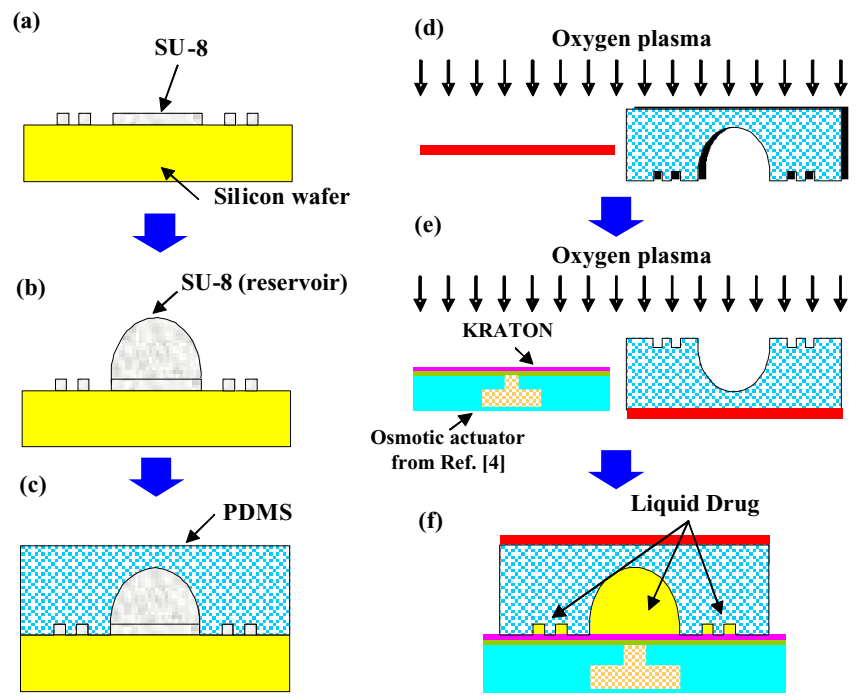

Figure 2. Fabrication process of the micro drug delivery system.

\section{FABRICATION PROCESS}

Figure 2 shows the fabrication process of the micro drug delivery system. A layer of $100 \mu \mathrm{m}$ thick negative photoresist (MicroChem SU-8 100) is spin-coated and patterned on top of a clean silicon wafer to create a mold for duplicating microfluidic components in the following polymer casting process as shown in Figure 2(a). Placing a droplet of SU-8 on top of the designated spot naturally forms the dome-shape mold of drug reservoir as shown in Figure 2(b). Because of its high viscosity, the SU-8 dome can be more than $1 \mathrm{~mm}$ tall and the actual height can be controlled by temperature. After the SU- 8 mold is fully UV cured, it is placed in a desiccator under vacuum for 2 hours with a vial containing a few drops of tridecafluoro-1,1,2,2-tetrahydrooctyl-1trichlorosilane to silanize the surfaces [6]. Silanization of the mold facilitates the removal of the polymeric replica after casting. Figure 3 shows the SEM micrograph of the SU- 8 mold where the drug reservoir is $1.8 \mathrm{~mm}$ in diameter and $800 \mu \mathrm{m}$ in height.

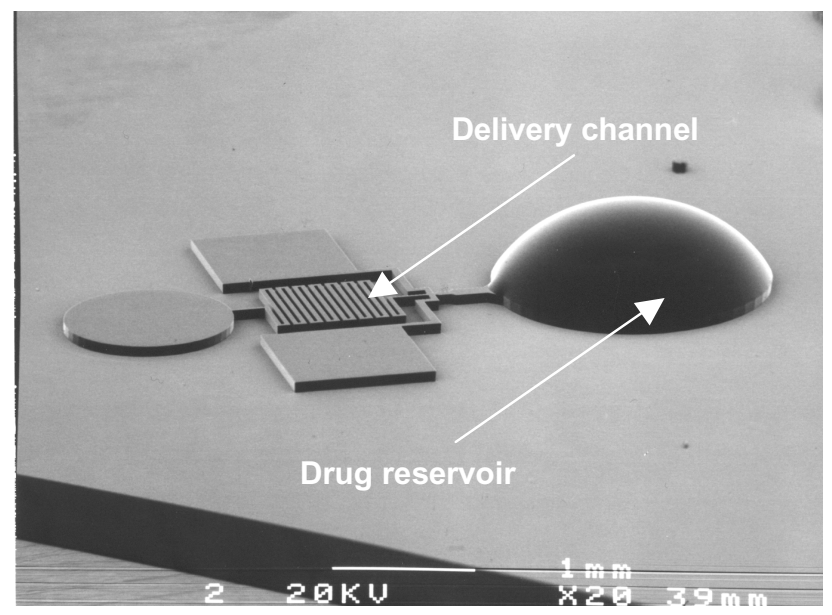

Figure 3. SEM micrograph of SU-8 mold for PDMS casting (process step b in Figure 2).

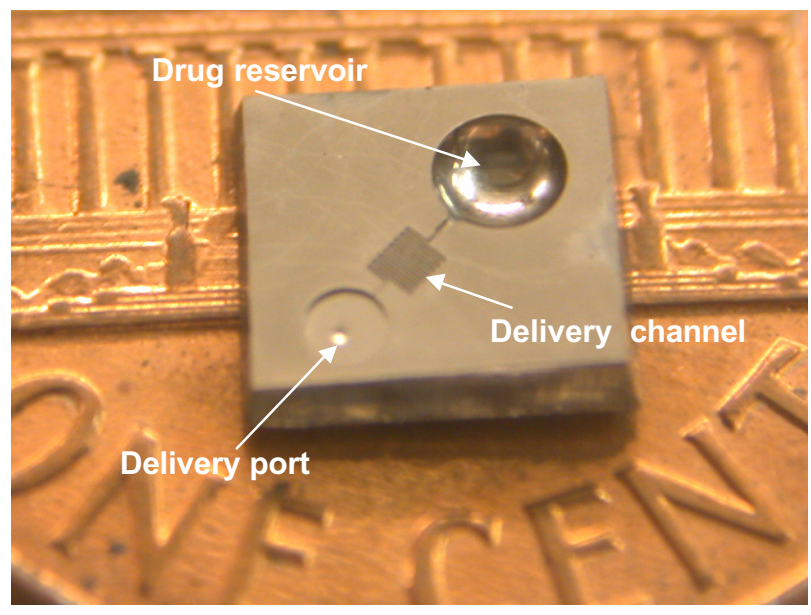

Figure 4. The water-powered drug delivery system pictured with a one-cent coin showing the drug reservoir, delivery channel, and delivery port.

A mixture of 10:1 PDMS prepolymer and curing agent (DowCorning Sylgard 184) is stirred thoroughly and then degassed under vacuum. The prepolymer and curing agent mixture is then poured onto the mold, degassed, and cured for 1 hour at $75^{\circ} \mathrm{C}$ as shown in Figure 2(c). Before PDMS is fully cured, a needle is inserted to form a delivery port (for flow rate measurement, not shown in the figure). After thoroughly curved, this PDMS replica is then peeled off from the mold and the needle is removed. Figure 4 is the molded PDMS replica containing microfluidic channel with a one-cent coin. Because PDMS is permeable to vapor, a $15 \mu \mathrm{m}$ thick barrier film (DuPont Mylar M45) is added on back of the replica as shown in Figure 2(d) to prevent liquid in the reservoir from evaporating and diffusing into outside environment. Native PDMS has low surface energy and cannot form strong enough bonding with Mylar. To prepare surfaces to achieve reliable bonding, both PDMS and Mylar surfaces are treated by oxygen plasma. These treated surfaces finally form strong bonding. 
Figure 5 shows the SEM micrograph of the bonding interface between PDMS and Mylar.

The major challenge of the integration process of a micro osmotic actuator (process details described previously [4]) and the PDMS replica is the bonding process. The top layer of the osmotic micro-actuator is a flexible and impermeable membrane made of vinylidene chloride and acrylonitrile copolymer (Dow Saran F-310) that cannot form strong bonding with PDMS even after plasma treatment [6]. For polymers cannot be directly bonded with PDMS by plasma treatment, we spin-coat an intermediate layer to facilitate bonding. Styrene-isoprene-styrene (S-I-S) copolymer (KRATON D-1193) is dissolved in toluene and spin-coated on top of the Saran membrane to form a $30 \mu \mathrm{m}$ thick intermediate layer. Both KRATON and PDMS are then exposed to oxygen plasma to activate the bonding surfaces (Fig. 2e) using a parallel-plate plasma etching system (Technics PE-IIA). Strong bonding can be achieved once two surfaces are brought to conformal contact. It has been reported that treated PDMS surface becomes hydrophilic and remains active for bonding if it is placed under liquid immediately after treatment [6,7]. Because the actuating membrane only moves in one direction, additional efforts are required to feed liquid drug into the drug reservoir and microfluidic channel. For aqueous drugs, since both treated surfaces are hydrophilic and the appearance of liquid around the bonding interface will not disturb the bonding process, liquid drug can fill microfluidic channel spontaneously and be encapsulated by employing the plasma-activated bonding process (Fig. 2f). The extra liquid drug in the gap between two bonding surfaces will eventually evaporate and strong bonding will be achieved once two surfaces are in contact. The cross-sectional view of the assembled micro drug delivery system is shown in Figure 6 where the thickness of the PDMS layer is $1.5 \mathrm{~mm}$.

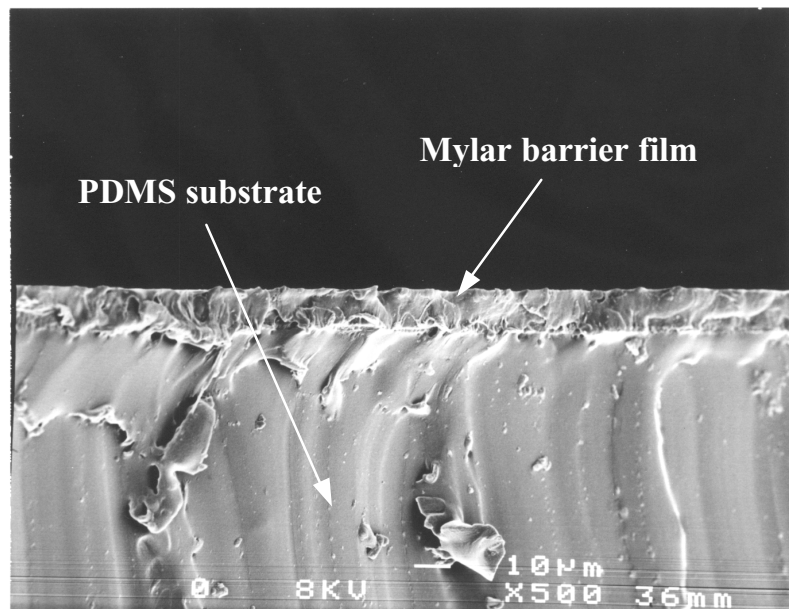

Figure 5. Cross-sectional SEM micrograph of the bonding interface between PDMS substrate and Mylar barrier film.

\section{MEASUREMENT RESULTS}

Flow rate measurement is conducted to verify the operation principles and design concepts. The released liquid flow from the fabricated micro drug delivery system is directed to a transparent polymer tube and the flow rate is estimated by tracing the movement of liquid-air interface in the tube. Polyimide tube of $310 \mu \mathrm{m}$ in outer diameter, $275 \mu \mathrm{m}$ in inner diameter, and $5 \mathrm{~cm}$ in length is inserted to the delivery port as shown in Figure 7 (the tube is cut to show its cross section). The gap between tube and delivery port is filled by pouring and curing additional PDMS prepolymer mixture around the connection. This will prevent the measurement error caused by leak. Another measurement error may come from the loss of liquid to outside environment by evaporation and diffusion. To estimate the loss of evaporation and diffusion along the tube, the following equation is used [8]:

$$
Q_{d v}=\frac{D_{v} P M_{w} A}{R_{0} T L} \ln \frac{P_{A_{2}}}{P_{A_{1}}}
$$

where $Q_{d v}$ is the rate of liquid loss by evaporation and diffusion, $D_{v}$ is the diffusivity of vapor along the tube, $P$ is the total pressure, $M_{w}$ is the molecular weight of liquid, $A$ is the cross-sectional area of the tube, $R_{0}$ is the universal gas constant, $T$ is the absolute temperature, $L$ is the length of tube, and $P_{A i}$ is the partial pressure of air at each end. Using tubes with small area to length ratios, the loss by evaporation and diffusion can be reduced. Considering water evaporating in a tube of $5 \mathrm{~cm}$ in length and $275 \mu \mathrm{m}$ in diameter at room temperature, this loss by evaporation and diffusion is $0.00256 \mu \mathrm{l} / \mathrm{hr}$, which is $1.3 \%$ of the overall convective flow rate. Figure 8 shows the measured total delivery volume with respect to time. The delivery rate is observed at $0.2 \mu 1 / \mathrm{hr}$ for an operation period of 10 hours.

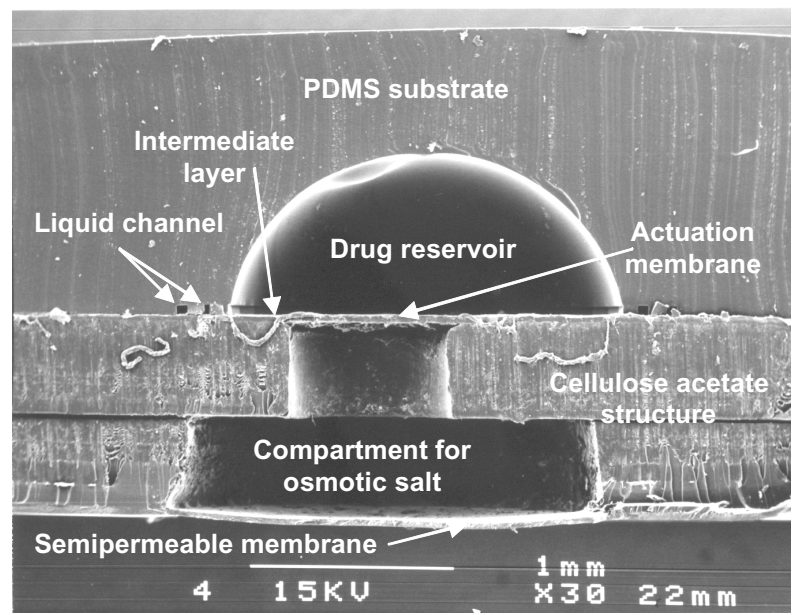

Figure 6. Cross-sectional SEM micrograph of the assembled micro drug delivery system.

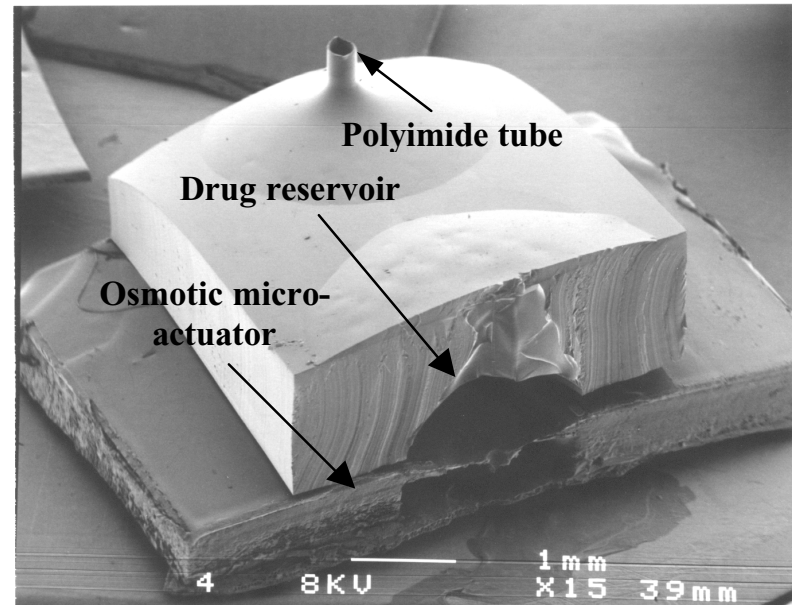

Figure 7. SEM micrograph of the micro drug delivery system with polyimide tube inserted for flow rate measurement. 


\section{DISCUSSIONS}

The length and cross-sectional area of the delivery channel are chosen to make sure that: (1) the diffusive flow rate of exiting drug is much lower than the convective flow rate of exiting drug such that the total drug delivery rate will keep consistent; and (2) the diffusive flow rate of entering fluid is much lower than the convective flow rate of exiting drug such that contamination, destabilizing, and diluting is minimized. To verify our current design, the following real values are used: $L=1 \mathrm{~cm}, Q_{c}=0.2 \mu \mathrm{l} / \mathrm{hr}$, $D=2 \times 10^{-10} \mathrm{~m}^{2} / \mathrm{s}, \mu=500$ centipoise, $w=30 \mu \mathrm{m}, h=100 \mu \mathrm{m}$, and $A=30 \mu \mathrm{m} \times 100 \mu \mathrm{m}$. Applying Equation 3 and 4, the calculation indicates that $I_{c}=0.00108$ and $\Delta P=873 \mathrm{~Pa}$. Even though the flow resistance across the delivery channel is high $\left(\sim 10^{16} \mathrm{~kg} / \mathrm{m}^{4} \mathrm{~s}\right)$, the resulted pressure drop is negligible compared to the driving pressure an osmotic actuator can provide because of the low drug flow rate.

Pressure drop will increase significantly if highly viscous drugs such as suspensions are delivered. Considering a suspension with viscosity $(\mu)$ equal to 50,000 centipoise being driven through the same microchannel with the same flow rate as the previous example, the pressure drop $\Delta P$ in this case will increase to $87 \mathrm{kPa}$ while $I_{c}$ remain around 0.001 . In order to keep the flow consistent and stable, it is desired to minimize both $I_{c}$ and $\Delta P$. For example, by increasing both cross-sectional area and length of the delivery channel to 4 times of the previous values, pressure drop will be reduced to $22 \mathrm{kPa}$ while $I_{c}$ remain the same value. For designing delivery channel in demand of transporting high viscosity drugs, another performance index can be defined as follows:

$$
I_{s}=\frac{I_{c}}{(\Delta P)^{-1}} \propto \frac{D \mu}{A}
$$

where $I_{s}$ defines the efficiency of simultaneously minimizing $I_{c}$ and $\Delta P$ and lower values indicate higher efficiency. With specified drug diffusivity and viscosity, cross-sectional area is the dominant factor for improving the overall performance considering both pressure drop and diffusive flow.

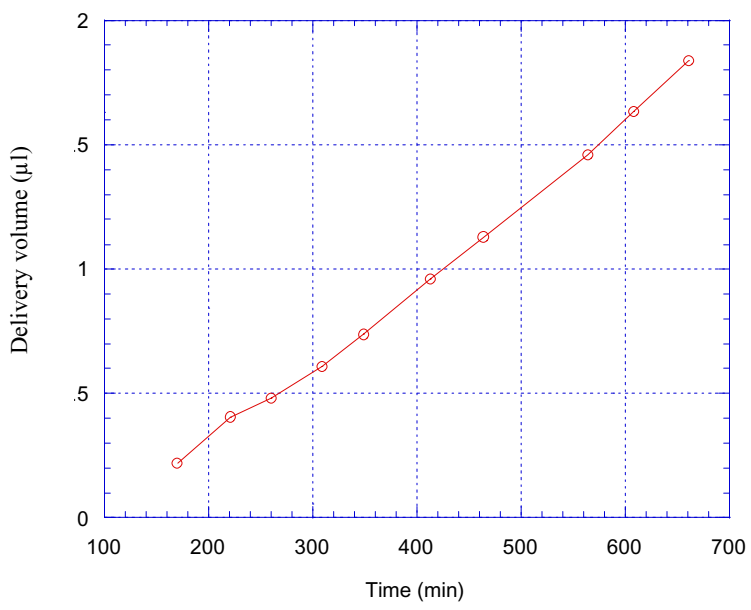

Figure 8. Measured delivery volume with respect to time of the micro drug delivery system.

Osmotic systems for the delivery of drugs to animals are well known. For implantation applications, larger drug reservoirs are preferred because their long working period will make replacement surgery less frequently. The current design of the micro drug delivery system can be modified to increase the delivery volume by increasing the area of the impermeable actuating membrane on top of the osmotic actuator as well as increasing the volume of the drug reservoir. The drug delivery rate can also be altered to be faster or slower by changing the area, thickness, and permeability of the semipermeable membrane. Biocompatibility, durability, reliability, and reactivity to drugs of the micro drug delivery system are major criteria for its potential implantation applications. Further investigation and improvement are required.

\section{CONCLUSIONS}

A plastic micro drug delivery system that draws power directly from water without any electrical power consumption is demonstrated. The system delivers liquid drugs with pressure up to $25 \mathrm{MPa}$ and the prototype design that utilizes a system volume of about $2.5 \times 2.5 \times 2 \mathrm{~mm}^{3}$ can deliver fluid drugs of $2 \mu \mathrm{l}$ with a constant delivery rate at $0.2 \mu \mathrm{l} / \mathrm{hr}$ for an operation period of 10 hours. Furthermore, the micro drug delivery system is made of biocompatible polymers including cellulose acetate and PDMS for biomedical applications. Three technical accomplishments have been achieved in this work: (1) bonding of PDMS with barrier polymers by introducing an intermediate elastomer layer for system integration; (2) direct liquid encapsulation and sealing at room temperature in the presence of water; and (3) delivery rates up to 20 times faster than previously reported results. In order to precisely control the drug delivery rate, it is found that the design of the drug delivery microchannel is critical and a performance index is proposed with respect to the cross-sectional area of the microchannel and the properties of the liquid drug.

\section{ACKNOWLEGEMENTS}

The authors would like to thank Alza, Dow, DuPont, and Kraton for providing testing samples. These devices are fabricated in the UC-Berkeley Microfabrication Lab. (silicon and SU-8 molds) and Microsystem Lab. (plastic parts). This work is supported in part by DARPA/MTO/BioFlips Grant F30602-00-20566 .

\section{REFERENCES}

1. C.L. Stevenson, F. Theeuwes, and J.C. Wright, "Osmotic Implantable Delivery Systems", Chapter 11, Handbook of Pharmaceutical Controlled Release Technology, Marcel Dekker, 2000.

2. U.S. Patent 6,156,331 (Dec. 5, 2000), Peery et al., (to Alza Corporation).

3. S. Venkatraman, N. Davar, A. Chester, and L. Kleiner, "An Overview of Controlled Release Systems", Chapter 22, Handbook of Pharmaceutical Controlled Release Technology, Marcel Dekker, 2000.

4. Y-C. Su, L Lin, and A.P. Pisano, "Water-Powered, Osmotic Microactuator", IEEE MEMS2001 Conference, pp. 393-396, 2001.

5. K. Foster and G.A. Parker, Fluidics: Components and Circuits, Wiley, 1970.

6. D.C. Duffy, J.C. Mcdonald, Olivier, J.A. Schueller, and G.M. Whitesides, "Rapid Prototyping of Microfluidic Systems in Poly(dimethylsiloxane)", Analytical Chemistry, Vol. 70, pp. 4974-4984, 1998.

7. B-H. Jo, L.M.V. Lerberghe, K.M. Motsegood, and D.J. Beebe, "Three-Dimensional Micro-Channel Fabrication in Polydimethylsiloxane (PDMS) Elastomer", IEEE Journal of Microelectromechanical Systems, Vol. 9, pp. 76-81, 2000.

8. J.P. Holman, Heat Transfer, McGraw-Hill, 1992. 